\section{Síndrome de Parsonage-Turner en paciente sometido a cirugía de revascularización miocárdica. Caso clínico}

\author{
GABRIEL CASTILLO ${ }^{1}$, JULIO SALAZAR ${ }^{2}$, \\ JUAN ESPINOZA ${ }^{3}$, NILO CARVAJAL ${ }^{3}$, \\ HERNÁN RUIZ ${ }^{3,4}$

\section{Parsonage-Turner syndrome. Report of one case}

We report a 59-year-old male who, three weeks after a coronary revascularization surgery, reported a sudden intense burning pain in his left upper limb. Two weeks later, he reports a paresis with difficulty to extend his left wrist and fingers. The electromyography showed a severe axonal damage of the radial nerve with distal denervation signs. This clinical picture probably corresponds to a neuralgic amyotrophy, an inflammatory disorder of the brachial plexus known by a number of terms, including Parsonage-Turner syndrome.

(Rev Med Chile 2019; 147: 1335-1339)

Key words: Brachial Plexus Neuritis; Coronary Artery Disease; Neuritis.
'Programa de Doctorado en Ciencias

Médicas y Especialidad. Escuela de

Postgrado. Facultad de Medicina.

Universidad de Chile. Santiago, Chile.

${ }^{2}$ Servicio de Neurología. Hospital San

José. Santiago, Chile.

${ }^{3}$ Servicio de Cardiología y Cardiocirugía.

Clínica Indisa. Santiago, Chile.

${ }^{4}$ Centro Cardiovascular. Hospital Clínico

San Borja-Arriarán. Santiago, Chile.

Trabajo no recibió financiamiento.

Los autores declaran no tener

conflictos de interés.

Recibido el 25 de junio de 2019, aceptado el 30 de octubre de 2019.

Correspondencia a:

Dr. Hernán Ruiz Pulido

Hospital Clínico San Borja-Arriarán.

Santiago, Chile.

hruizpulido@mi.cl

Dr. Gabriel Castillo Rozas

Facultad de Medicina Universidad de

Chile. Santiago, Chile

gabrielcastillor@ug.uchile.cl
L as complicaciones neurológicas asociadas a la cirugía de revascularización miocárdica (CRM) pueden llegar hasta 60\%, siendo la mayoría de ellas leves y autolimitadas. Menos de $2 \%$ generarían secuelas invalidantes ${ }^{1}$. Cerca de $12 \%$ corresponderían a afecciones del sistema nervioso periférico (SNP), dentro de las cuales destacan la plexitis y las neurapraxias que afectan a los miembros superiores.

\section{Caso clínico}

Paciente de 59 años, con antecedentes de tabaquismo (paquete-año 45), hipertensión arterial y dislipidemia. Acudió a un servicio de urgencias en abril de 2017 por cuadro de dolor retroesternal opresivo de inicio brusco irradiado a brazo izquierdo y asociado a síntomas neurovegetativos, con electrocardiograma compatible con infarto agudo de miocardio con supradesnivel ST.
Abandonó el hospital mientras se programaba la coronariografía.

Consultó nuevamente 3 días más tarde en Clínica Indisa, sin dolor. El electrocardiograma (ECG) mostraba taquicardia sinusal, extrasístoles ventriculares aisladas y signos de infarto en evolución. En coronariografía se evidenció enfermedad grave de tres vasos, por lo que se decidió resolución quirúrgica.

$\mathrm{Al} 4^{\circ}$ día postinfarto se realizó cirugía de revascularización miocárdica (CRM) con puente de la arteria mamaria interna izquierda a la descendente anterior y venoso secuencial a la coronaria derecha distal y a la segunda obtusa marginal, sin incidentes. Fue dado de alta al $7^{\circ}$ día postoperatorio en buenas condiciones generales y con terapia de prevención secundaria de cardiopatía coronaria.

Presentó tres semanas luego de la cirugía cuadro de inicio súbito, durante la madrugada, de dolor urente en extremidad superior izquierda, difuso, referido como "el peor dolor de la vida, 
como si me cortaran el brazo", no asociado a precordalgia, con respuesta parcial a analgesia. Consultó en servicio de urgencia, sin angina, sin

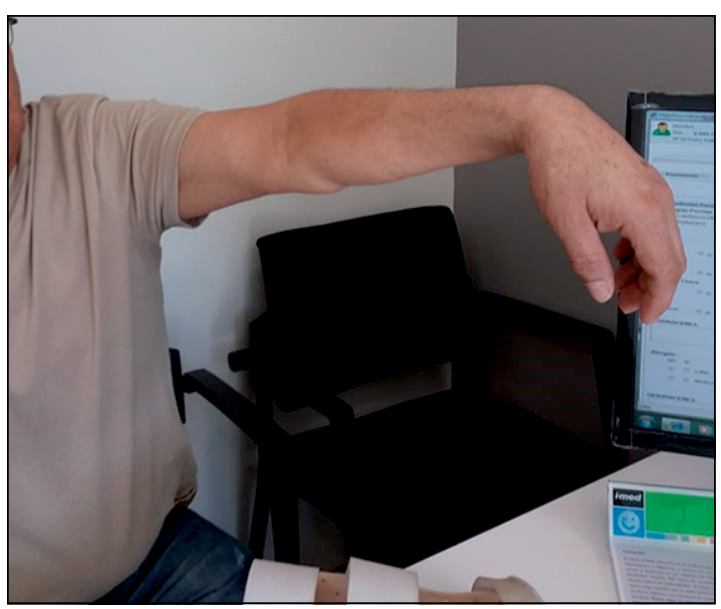

Figura 1. Se observa "parálisis en gota" de mano izquierda, dos semanas tras el inicio del cuadro álgico. Fuerza evaluada como M1 (mayo 2017). Foto autorizada por paciente para divulgación científica. disnea. ECG y laboratorio normales. Se interpretó como cuadro musculoesquelético.

Dos semanas más tarde, se agregó paresia en extensión de muñeca y dedos de mano izquierda (Figura 1), por lo que fue derivado a Neurología. En la evaluación neurológica, se constató paresia de extensión de dedos y muñeca M1-M2 de extremidad superior izquierda, con hipotonía leve, reflejo de Hoffman ausente, sin déficit sensitivo. Se interpretó como mononeuritis de nervio radial, por lo que se solicitó perfil inmunorreumatológico, que resultó negativo (hemograma, PCR, VHS, perfil ENA de anticuerpos, anticuerpos anti-DNA, antimieloperoxidasa y antiproteinasa 3). Electromiografía con signos de daño axonal grave de nervio radial/interóseo posterior y signos denervatorios distales. Se comprobó compromiso neuropático crónico de territorios C5 y C6. La tomografía computada (TAC) de encéfalo no mostró lesiones. Se decidió mantener en observación, constatándose mejoría progresiva en función motora durante el seguimiento. El paciente abandonó controles neurológicos. Asistió a control cardiológico a los 24 meses de seguimiento, evidenciándose

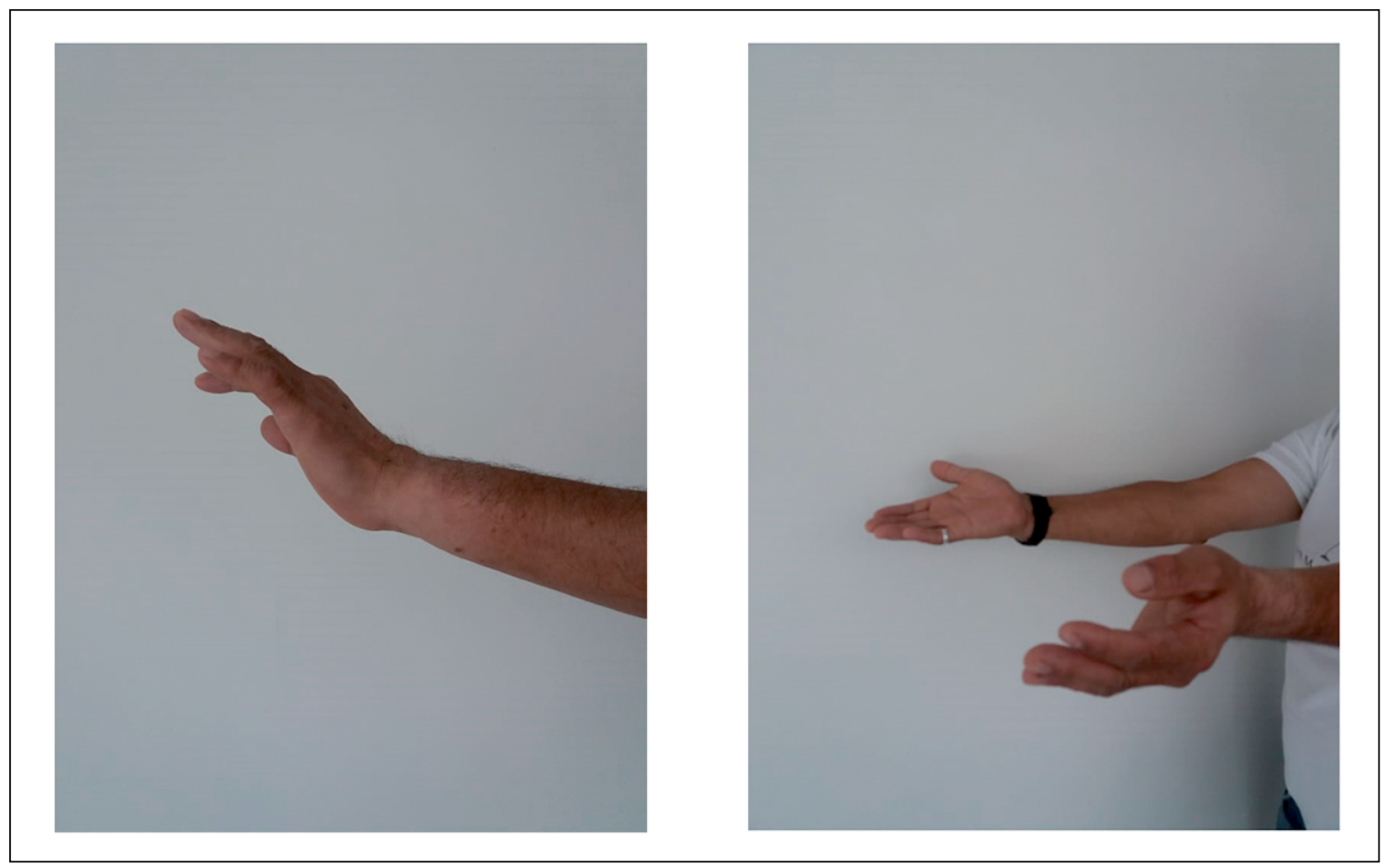

Figura 2. En comparación a imagen anterior, se observa mejoría en fuerza (M4+), con déficit neurológico residual (enero 2019). Foto autorizada por paciente para divulgación científica. 
paresia residual M4+ para extensión de muñeca izquierda y M4 para extensión de dedos (Figura 2) y parche hipoestésico en relación a primer dedo izquierdo.

\section{Discusión}

Dentro las afecciones del SNP post-CRM, el daño al plexo braquial puede llegar hasta $7 \%{ }^{1}$. Las teorías que se han planteado para explicarlas son fundamentalmente los factores mecánicos y los factores inflamatorios. En relación con el primero, se generaría daño indirecto sobre las raíces nerviosas inferiores del plexo braquial por la tracción esternal ${ }^{2}$, así como por la posición de los brazos durante la cirugía y la extracción de la arteria mamaria interna ${ }^{3}$. Se ha visto que el daño al plexo braquial sería diez veces menos frecuente en aquellas cirugías donde no se extrae la arteria mamaria interna ${ }^{3}$. En los reportes de casos donde el daño nervioso se ha atribuido a factores mecánicos, el déficit neurológico se instala precozmente durante el postoperatorio ${ }^{1}$.

Dentro de las entidades inflamatorio-inmunológicas destaca la neuralgia amiotrófica (NA) (acuñada a mediados del siglo XX como síndrome de Parsonage-Turner) que corresponde a una neuropatía de probable origen inflamatorio y que se ha asociado a múltiples precipitantes: trauma, parto, cirugía, entre otros factores ${ }^{4}$, que suelen anteceder a la aparición del cuadro en 2-4 semanas. Se caracteriza por dolor agudo e intenso, seguido por paresia y atrofia muscular.

El cuadro clásico corresponde a un paciente que se despierta súbitamente durante la noche por dolor en el hombro o brazo, de gran intensidad y de difícil tratamiento. Luego de algunos días se agrega paresia que afecta típicamente al nervio torácico largo, supraescapular o interóseo anterior, con sensación de hormigueo en el territorio radial o del nervio cutáneo antebraquial lateral y un parche de hipoestesia en la cara lateral del brazo, según territorio del nervio axilar. El cuadro de dolor intenso suele durar 2-3 semanas, y puede enmascarar la sintomatología neurológica, que puede no tener recuperación ${ }^{4}$. En un tercio de los pacientes, el cuadro clínico se manifiesta a través del compromiso de otros nervios. Se han descrito casos de compromiso lumbosacro e incluso del nervio frénico, en pacientes con disnea inexplicada ${ }^{5-6}$ (Tabla 1).
La incidencia actual se estima en 1 en $1.000 \mathrm{al}$ año, muy superior a lo publicado hace 30 años, cuando se estimaba en $1-3$ en 100.000 por año ${ }^{6,7}$. Predomina en hombres en una relación 2:1 y su edad media de presentación varía según el tipo: 25 años en formas hereditarias y 40 años en las formas idiopáticas. La recurrencia puede ascender hasta $75 \%$ en las formas hereditarias y $25 \%$ en las formas idiopáticas.

La fisiopatología exacta es desconocida, pero se plantea que correspondería a la conjunción de múltiples factores: predisposición genética, factores biomecánicos y fenómenos de autoinmunidad. Si bien no se han podido identificar mutaciones en los casos de NA idiopática ${ }^{9}$, se han documentado mutaciones en los genes SEPT9 en las formas hereditarias ${ }^{10}$, en cuyos casos hay mayor riesgo de recurrencia y de compromiso nervioso extrabraquial. Por otro lado, no se ha demostrado vulnerabilidad inmunológica, pero la mitad de los afectados han sido expuestos a distintas noxas inmunológicas: infecciones, cirugía, parto y estrés físico o mental ${ }^{11}$. Existen reportes de NA asociado a virus de la hepatitis E (VHE) ${ }^{12}$, Bartonella henselae $^{13}$, Borrelia burgdorferi e incluso a virus de la inmunodeficiencia humana (VIH). Pese a lo anterior, la hipótesis de un origen inmunome-

Tabla 1. Distribución relativa de afectación nerviosa en pacientes con neuralgia amiotrófica

\begin{tabular}{|lc|}
\hline Nervio involucrado & Frecuencia \\
\hline Supraescapular & $23 \%$ \\
\hline Torácico largo & $16 \%$ \\
\hline Ramos motores del plexo braquial & $14 \%$ \\
\hline Interóseo anterior & $11 \%$ \\
\hline Axilar & $10 \%$ \\
\hline Musculocutáneo & $6 \%$ \\
\hline Radial & $5 \%$ \\
\hline Mediano & $3 \%$ \\
\hline Frénico & $2 \%$ \\
\hline Cutáneo antebraquial lateral & $2 \%$ \\
\hline Espinal accesorio & $1 \%$ \\
\hline Interóseo posterior & $1 \%$ \\
\hline Ulnar & $1 \%$ \\
\hline Facial & $0,1 \%$ \\
\hline
\end{tabular}

Adaptado de Clinical Neurophysiology 2009; 120(2). DOI:10.1016/j.clinph.2008.10.073. 
diado se apoya en hallazgos histopatológicos de infiltrado perineural de linfocitos T y macrófagos, sin hallazgos de inflamación perivascular ${ }^{14}$. Se ha documentado también la presencia de linfocitos B CD20+ y macrófagos CD68+ en tejido perineural con fibrosis y degeneración axonal, así como disminución en sangre periférica de linfocitos $\mathrm{T}$ supresores, como se observa en pacientes con síndrome de Guillain-Barré. Así, se ha planteado que los factores biomecánicos producirían microdisrupciones de la barrera hematonerviosa, permitiendo la exposición de antígenos que son reconocidos erróneamente por el sistema inmune y permitiendo así el montaje de una respuesta contra autoantígenos e iniciándose la cascada de eventos que lleva a la degeneración axonal difusa y que no se corresponde clínicamente con una localización específica ${ }^{11}$.

El diagnóstico es clínico. La anamnesis es clara en la mayoría de los casos, así como el examen físico que suele tener hallazgos característicos y cuyos detalles se escapan al objetivo de este manuscrito. El problema con el diagnóstico está relacionado con el bajo nivel de sospecha y con el bajo rendimiento que tiene el laboratorio complementario ${ }^{15}$. No obstante, algunas claves semiológicas que permiten diferenciar el cuadro con tendinopatías, bursitis o cervicobraquialgias es la ausencia de un patrón radiculopático ni neuropático claro.

Lamentablemente, las pruebas diagnósticas poseen un bajo rendimiento; si bien la electromiografía es una técnica sensible para la detección de lesiones axonales y desmielinizantes, el rendimiento es bajo durante las primeras semanas de evolución de la enfermedad ${ }^{16}$. Por otro lado, la ecografía torácica es útil en afección del nervio frénico, al poder visualizar la parálisis diafragmática $^{17}$ y la neurografía por resonancia magnética ha mostrado ser más sensible que la resonancia magnética convencional para el diagnóstico de lesiones que afecten al SNP; característicamente, se evidencia engrosamiento e hiperintensidad del nervio o plexo afectado durante la fase aguda de la enfermedad ${ }^{18}$.

En relación al tratamiento, la analgesia es primordial: lo recomendado sería una combinación de antiinflamatorios no esteroidales con opiáceos $^{8}$. Si bien se ha podido documentar el rol de los fenómenos inmunomediados en la NA, el uso de corticoides, inmunoglobulinas e inmuno- terapia no han logrado demostrar beneficios en estudios con distribución aleatoria; en un estudio open-label retrospectivo, el uso de prednisona oral durante 1 mes se asoció a buen manejo del dolor y a satisfactoria recuperación durante la fase crónica de la enfermedad ${ }^{19}$. Por otro lado, existe una baja derivación hacia servicios de fisiatría, y muchas derivaciones son realizadas precozmente, aun cuando los pacientes cursan con importante debilidad muscular y dolor.

Existe evidencia disímil en relación a la recuperación funcional a largo plazo, con reportes que llegan a 10\% de recuperación funcional completa a un seguimiento de 3 años ${ }^{11}$. En un seguimiento de cohortes basado en un cuestionario aplicado a 248 pacientes con NA, más de la mitad de los pacientes tenía limitaciones diarias por dolor y en el grupo que no sufría dolor, $60 \%$ presentaba una paresia residual ${ }^{20}$.

En relación al caso clínico, la forma de presentación clínica, el tiempo entre la cirugía y el inicio de los síntomas y la evolución posterior orientan fuertemente a una etiología inflamatoria-inmunológica que se corresponde con un síndrome de Parsonage-Turner o neuralgia amiotrófica. Según nuestro conocimiento, el caso clínico presentado correspondería al primer reporte en Chile de síndrome de Parsonage-Turner con afectación radial tras una cirugía de revascularización miocárdica.

\section{Referencias}

1. Chong AY, Clarke CE, Dimitri WR, Lip GY. Brachial plexus injury as an unusual complication of coronary artery bypass graft surgery. Postgrad Med J 2003; 79: 84-6.

2. Fernández DC, Duarte J, Lozano A, Torrente N. [Radial nerve injury caused by external compression during the dissection of the internal mammary artery in coronary surgery]. Rev Esp Anestesiol Reanim 1992; 39: 371-3.

3. Vahl CF, Carl I, Muller-Vahl H, Struck E. Brachial plexus injury after cardiac surgery. The role of internal mammary artery preparation: prospective study on 1000 consecutive patients. J Thorac Cardiovasc Surg 1991; 102: 724-9.

4. Van Eijk J, Groothuis JT, Van Alfen N. Neuralgic amyotrophy: an update on diagnosis, pathophysiology, and treatment. Muscle Nerve 2016; 53: 337-50.

5. Byrne E. Extended neuralgic amyotrophy syndrome. Aus NZ J Med 1987; 17: 34-8. 
6. England JD, Summer AJ. Neuralgic amyotrophy: an increasingly diverse entity. Muscle Nerve 1987; 10: 60-8.

7. MacDonald BK, Cockerell OC, Sander JW, Shorvon SD. The incidence and lifetime prevalence of neurological disorders in a prospective community-based study in the UK. Brain 2000; 123: 665-76.

8. Van Alfen N, van Eijk JJ, Ennik T, Flynn SO, Nobacht IE, Groothuis JT, et al. Incidence of neuralgic amyotrophy (Parsonage Turner Syndrome) in a primary care setting - a prospective cohort study. PLoS One 2015; 10 (5): e0128361.

9. Van Alfen N. Clinical and pathophysiological concepts of neuralgic amyotrophy. Nat Rev Neurol 2011; 7: 31522.

10. Van Alfen N, Hannibal MC, Chance PF, Van Engelen BG. Hereditary Neuralgic Amyotrophy. In: Adam MP, Ardinger HH, Pagon RA, et al., editors. GeneReviews ${ }^{\circledR}$ [Internet]. Seattle (WA): University of Washington, Seattle; 1993-2019. Disponible en: https://www.ncbi.nlm. nih.gov/books/NBK1395/ [Consultado el 20 de abril de 2019].

11. Van Alfen N, van Engelen BG. The clinical spectrum of neuralgic amyotrophy in 246 cases. Brain 2006; 129: 438-50.

12. Dartevel A, Colombe B, Bosseray A, Larrat S, Sarrot-Reynauld F, Belbezier A, et al. Hepatitis E and neuralgic amyotrophy: five cases and review of literature. J Clin Virol 2015; 69: 156-64.

13. Stek CJ, van Eijk JJ, Jacobs BC, Enting RH, Sprenger
HG, van Alfen N. Neuralgic amyotrophy associated with Bartonella henselae infection. J Neurol Neurosurg Psychiatry 2011; 82: 707-8.

14. Arts WF, Busch HG Van der Brand HJ, Jennekens FG, Frants RR, Stefanko SZ. Hereditary neuralgic amyotrophy. Clinical, genetic, electrophysiological and histopatological studies. J Neurol Sci 1983; 62: 261-79.

15. Van Alfen N, Malessy MJ. Diagnosis of brachial and lumbosacral plexus lesions. Handb Clin Neurol 2013; 115: 293-310.

16. Feinberg JH, Doward DA, Gonsalves A. Cervical radiculopathy vs Parsonage-Turner Syndrome: a case report. HSSJ 2007; 3: 106-11.

17. Boon AJ, Sekiguchi H, Harper CJ, Strommen JA, Ghahfarokhi LS, Watson JC. Sensitivity and specificity of diagnostic ultrasound in the diagnosis of phrenic neuropathy. Neurology 2014; 83: 1264-70.

18. Duman I, Guvenc I, Kalyon TA. Neuralgic amyotrophy, diagnosed with magnetic resonance neurography in acute stage: a case report and review of the literature. Neurologist 2007; 13: 219-21.

19. Van Alfen N, van Engelen BG, Hughes RA. Treatment for idiopathic and hereditary neuralgic amyotrophy (brachial neuritis). Cochrane Database Syst Rev 2009b; (3): CD006976.

20. Cup EH, Ijspeert J, Janssen RJ, Bussemaker-Beumer C, Jacobs J, Pieterse AJ. Residual complains after neuralgic amyotrophy. Arch Phys Med Rehabil 2013; 94: 67-73. 